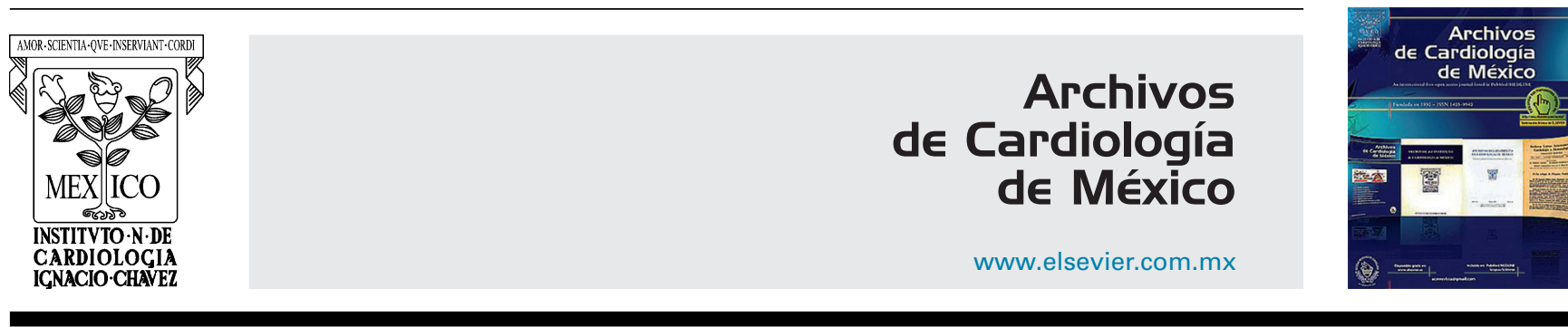

INVESTIGACIÓN BÁSICA

\title{
Aumento en la efectividad de la identificación de cimas y pies en el pulso fotopletismográfico al reconstruirlo mediante filtrado adaptativo
}

\author{
Brayans Becerra-Luna ${ }^{a}$, Raúl Martínez-Memije ${ }^{\mathrm{a}}$, Raúl Cartas-Rosado ${ }^{\mathrm{b}}$ \\ y Oscar Infante-Vázquez ${ }^{a, *}$
}

a Departamento de Instrumentación Electromecánica, Instituto Nacional de Cardiología Ignacio Chávez, México, D.F., México

b Consejo Nacional de Ciencia y Tecnología, Ciudad de México, México

Recibido el 13 de abril de 2016; aceptado el 4 de octubre de 2016

\author{
PALABRAS CLAVE \\ Algoritmo de mínimos \\ cuadrados \\ promediado; \\ Pulso \\ fotopletismográfico; \\ Acelerómetro; \\ Filtros adaptativos; \\ Artefactos por \\ movimiento
}

\begin{abstract}
Resumen
Objetivo: Mejorar la identificación de cimas y pies en el pulso fotopletismográfico (PPG, por sus siglas en inglés), deformado por efecto del ruido miocinético, mediante la implementación de un dedal modificado y filtrado adaptativo.

Método: Se obtuvo el PPG en 10 voluntarios sanos empleando 2 sistemas de fotopletismografía colocados en el dedo índice de cada mano, y registrándolos simultáneamente durante 3 min. Durante el primer minuto de registro, ambas manos estuvieron en reposo, y durante los 2 min posteriores, solo la mano izquierda realizó movimientos cuasi-periódicos para añadir ruido miocinético. Se emplearon 2 metodologías para procesar las señales fuera de línea, en una se usó un filtro con el algoritmo de mínimos cuadrados promediados (LMS, por sus siglas en inglés) y en la otra se hizo un preprocesamiento adicional al filtrado LMS. Ambas metodologías fueron comparadas y la de menor error porcentual en la señal recuperada se utilizó para valorar la mejora en la identificación de cimas y pies del PPG.

Resultados: El error promedio obtenido fue del $22.94 \%$ para la primera metodología, y del $3.72 \%$ para la segunda. Los errores en la identificación de cimas y pies antes de filtrar el PPG fueron del 24.26 y $48.39 \%$, respectivamente, una vez filtrados, disminuyeron a 2.02 y $3.77 \%$, respectivamente.

Conclusiones: El filtrado adaptativo basado en el algoritmo LMS, más una etapa de preprocesamiento, permite atenuar el ruido miocinético en el PPG, y aumentar la efectividad en la
\end{abstract}

\footnotetext{
* Autor para correspondencia. Departamento de Instrumentación Electromecánica, Instituto Nacional de Cardiología Ignacio Chávez, Juan Badiano 1, Col Sección 16, Del. Tlalpan; México, D.F. 14080, México; Teléfono: +52 555573 2911; Extensión; 1386 ; Fax: +52 55 5573 0926.

Correo electrónico: infosc@cardiologia.org.mx (0. Infante-Vázquez).
} 
identificación de cimas y pies de pulso, que resultan de gran importancia para una valoración médica.

(C) 2016 Instituto Nacional de Cardiología Ignacio Chávez. Publicado por Masson Doyma México S.A. Este es un artículo Open Access bajo la licencia CC BY-NC-ND (http://creativecommons. org/licenses/by-nc-nd/4.0/).

\section{KEYWORDS}

Least mean square algorithm; Photoplethysmographic pulse signal; Accelerometer; Adaptive filters; Motion artifacts

\section{Introducción}

Actualmente, la monitorización ambulatoria de signos vitales, como parte de las tendencias del autocuidado, ha tomado un fuerte auge con la integración de sistemas médicos a los dispositivos móviles y su conectividad a Internet ${ }^{1,2}$, lo que ha generado, a su vez, una mayor demanda de sistemas portables que sean capaces de medir los signos vitales de manera confiable. Las técnicas y métodos empleados en esta tendencia son diversos, y preferentemente no invasivos, debido a que la aplicación final es el automonitoreo ${ }^{3}$.

La fotopletismografía es una técnica ampliamente usada para medir parámetros fisiológicos de manera no invasiva, que está basada en un sistema optoelectrónico ${ }^{4}$ formado por un diodo emisor de luz (light emitting diode [LED]) y un elemento receptor (fototransistor), que en conjunto se encargan de iluminar la piel, y detectar las variaciones lumínicas que se producen debido a los efectos de reflexión y absorción de la luz por la sangre en las arterias, principalmente. La fotopletismografía permite así visualizar los cambios de volumen sanguíneo dados por la actividad cardiaca, al registrar la onda de pulso sanguíneo, a partir de la cual se puede obtener información de variables relacionadas con el sistema cardiovascular como: frecuencia cardiaca, tensión arterial, oximetría de pulso y frecuencia respiratoria.

Los registros obtenidos a partir de esta técnica son sensibles, y están limitados por factores como el correcto posicionamiento de los dispositivos optoelectrónicos y la presión ejercida en el dedal al comprimir las arterias. Otra limitación es el ruido inducido por los movimientos que se generan en la extremidad donde se realiza la toma del pie en el pulso fotopletismográfico (PPG), ya sea por dificultades debidas a alguna enfermedad ${ }^{5}$ asociada a daño neurodegenerativo como el mal de Parkinson, u otros artefactos de movimiento $^{6}$. El PPG también puede estar contaminado por interferencia de la línea eléctrica de alimentación principal.

La morfología de la señal del PPG contiene información de interés clínico ${ }^{7}$, donde los médicos buscan factores de riesgo cardiovascular ${ }^{8}$, y pueden realizar una valoración del estado de salud del paciente, por lo que es muy importante poder registrar dicha señal con la menor deformación posible.

Una forma de minimizar algunas de las dificultades que se presentan al registrar señales del PPG es procesarlas mediante filtros de coeficientes fijos; sin embargo, estos filtros tienen a su vez otro conjunto de limitaciones, dado que algunos de los problemas de adquisición anteriormente 
mencionados como el ruido miocinético, provocan en la señal del PPG una deformación tal que la información de interés se encuentra mezclada con el ruido que se genera por el fenómeno de movimiento, imposibilitando la extracción correcta de la información de interés. Para mejorar el proceso de filtrado bajo las condiciones anteriormente mencionadas, existen otro tipo de filtros que son adaptativos. La principal ventaja de los filtros adaptativos es que los coeficientes que lo describen se ajustan a la dinámica de la señal que se desea procesar. Los filtros adaptativos han sido utilizados con configuración de canceladores de ruido en el procesamiento de señales del ECG ${ }^{9}$ y del PPG ${ }^{10-12}$. En estas aplicaciones se usaron estructuras de filtrado de gran carga computacional, que en el caso del tratamiento del ECG, la finalidad fue atenuar la influencia de la señal respiratoria en la variabilidad de la frecuencia cardiaca ${ }^{13}$.

En este trabajo se propone aumentar la efectividad en la identificación de los puntos notables (cimas y pies) del PPG, al atenuar el ruido miocinético que contamina a los registros del PPG, buscando así mejorar tanto la calidad como la confiabilidad de dichas señales. Para lograr el objetivo se usó una etapa de preprocesamiento además de un filtro adaptativo en configuración de cancelador de ruido (adaptive noise canceller $[\mathrm{ANC}])^{14}$ compuesto por un filtro digital de respuesta finita (finite impulse response [FIR]) y un algoritmo de adaptación denominado de mínimos cuadrados promediados (least mean square [LMS]) ${ }^{15}$, manteniendo así una estructura de menor carga computacional comparada con los trabajos antes mencionados.

\section{Métodos}

Para este trabajo se considera que existe una relación de tipo lineal entre el PPG, sin movimiento, y el que ha sido deformado por el movimiento miocinético, por lo que utilizamos una herramienta de filtrado lineal denominada filtro adaptativo, el cual se compone en este caso de un filtro digital tipo FIR y un algoritmo de adaptación LMS.

\section{Descripción de sujetos voluntarios}

El protocolo bajo el que se llevó acabo el registro de las señales de la fotopletismografía ha sido revisado y aprobado por el Comité de Ética del Instituto Nacional de Cardiología «lgnacio Chávez», y está identificado con la clave 13-820.

Los criterios de inclusión fueron: tener menos de 60 años a la fecha del registro del PPG, y haber sido diagnosticado como sujeto clínicamente sano por un médico. Dentro de los criterios de exclusión estuvieron el tener una edad superior a 60 años a la fecha del registro, tener padecimientos de tipo cardiovascular y/o neurológico, y haber sido diagnosticado con algún padecimiento degenerativo que obstruya el desarrollo del protocolo como diabetes o daño renal.

Los criterios de eliminación de los registros fueron: contaminación del PPG por fuentes distintas al movimiento miocinético y al efecto del ruido de línea $(60 \mathrm{~Hz})$, pobre relación señal a ruido en el cual la amplitud en la señal sea mucho menor que el nivel de ruido al que es sometido, y que no mantengan una correlación mayor a 0.5 entre las señales del PPG y las registradas por el acelerómetro (Acc, por sus siglas en inglés).
Previo al registro del PPG de cada uno de los participantes, se les informó sobre el procedimiento a seguir, y posterior a ello, se les solicitó firmar la carta de consentimiento informado.

Este protocolo es considerado un estudio piloto, por lo que la muestra de voluntarios que participaron fue de 10 sujetos sanos, 8 del sexo masculino y 2 del sexo femenino. Los voluntarios fueron valorados clínicamente por medio de interrogatorio y auscultación por un médico.

La edad promedio de la muestra fue de 31.6 años (en un rango de 22 a 58), siendo 7 de los participantes menores de 30 años. La consideración de invitar a participar a sujetos, en su mayoría jóvenes, se fundamenta en el hecho de obtener registros que estén deformados mayormente por el ruido miocinético, y no por algunas otras dificultades asociadas a enfermedades como la rigidez arterial ${ }^{16}$, dado que a mayor edad existen mayores probabilidades de deformación en la morfología del $\mathrm{PPG}^{7}$, no solo por ruido miocinético.

\section{Descripción del sistema para registros del pulso fotopletismográfico}

Para el registro de señales del PPG y del movimiento miocinético de los voluntarios se utilizó un sistema como el que se indica en el diagrama a bloques de la figura 1, el cual muestra las partes más significativas del sistema:

\section{Características eléctricas de los sistemas de registro}

Para el registro de señales del PPG se diseñaron 2 equipos de fotopletismografía con características eléctricas semejantes en voltaje de operación, ancho de banda, ganancia y sensibilidad (tabla 1). Cada equipo cuenta con una etapa seguidora de voltaje, 2 etapas de amplificación en voltaje y una sección de filtrado analógico tipo Butterworth de 3. ${ }^{\mathrm{er}}$ orden. El diseño de los equipos de fotopletismografía ha sido caracterizado y ajustado en trabajos previos ${ }^{10}$, de acuerdo a los estándares aceptados para esta aplicación ${ }^{4}$. La diferencia entre ambos equipos es que en uno de ellos se incluyó un Acc, que además permite registrar movimiento en los 3 ejes coordenados, el cual se limitó en ancho de banda usando un filtro pasa bajas a $45 \mathrm{~Hz}$ para cada uno de sus ejes. Dicho Acc permite registrar cambios de aceleración de $\pm 1.5 \mathrm{~g}$, siendo $\mathrm{g}$ la constante de aceleración de la gravedad que es de $9.81 \mathrm{~m} / \mathrm{s}^{2}$.

\section{Adquisición de señales}

Para la digitalización del PPG y del movimiento se utilizó una tarjeta de adquisición de datos modelo USB1-208FS de Measurement Computing junto con el software Trace DAQ ${ }^{\circledR}{ }^{17}$. La tarjeta cuenta con un convertidor analógico/digital (A/D) a 11 bits de resolución, su comunicación es por interfaz USB, y adquiere datos con una frecuencia de muestreo de 100 muestras/segundo. El tiempo de registro simultáneo en ambos fotopletismógrafos fue de $3 \mathrm{~min}$ para cada uno, y los registros se almacenaron en archivos de texto. 


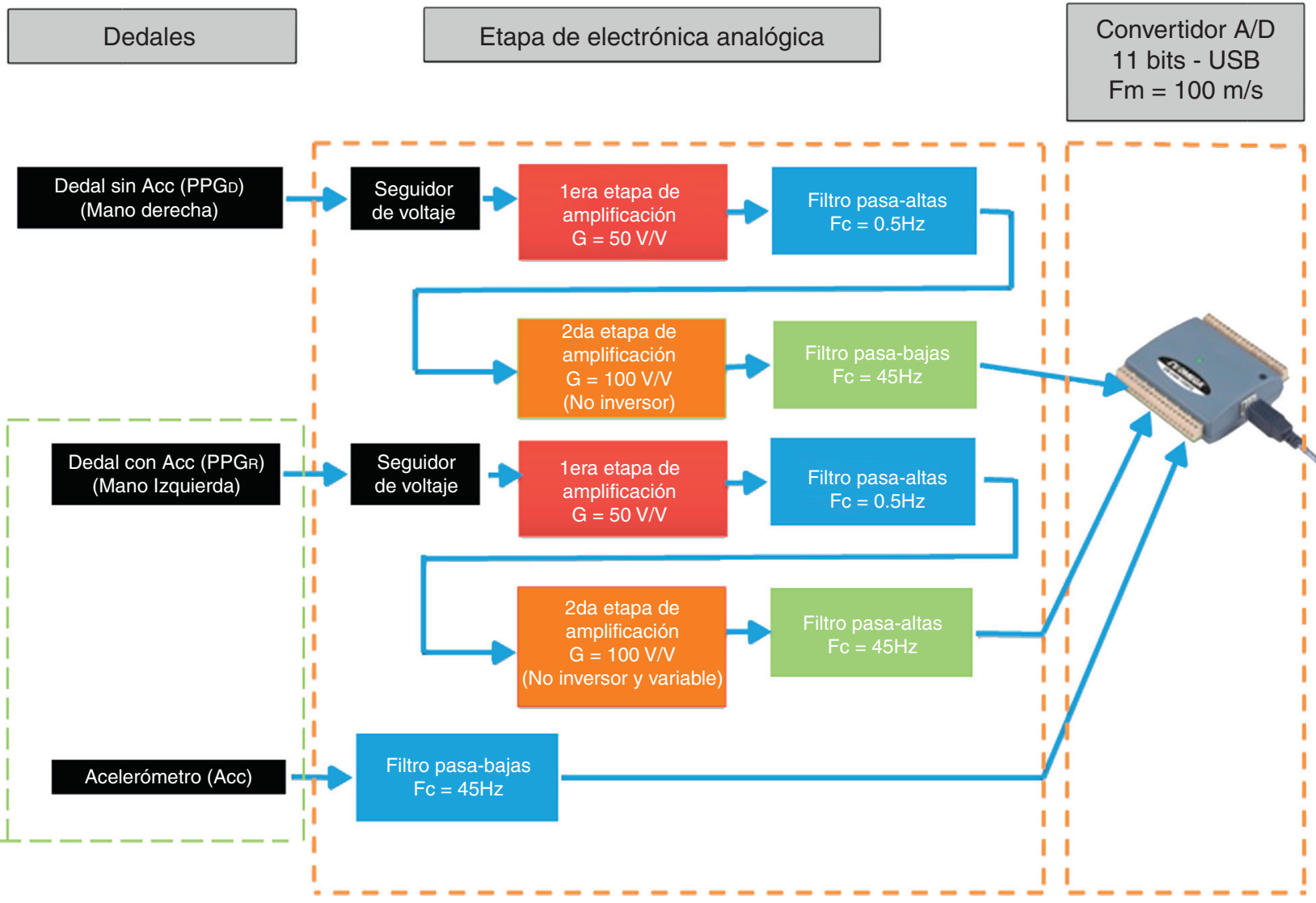

Figura 1 Diagrama a bloques del sistema para el registro de señales del PPG y movimiento mediante un Acc, caracterizando las partes principales que lo componen. $F m$ indica frecuencia de muestreo, $\mathrm{m} / \mathrm{s}$ es muestras por segundo y $A / D$ se refiere a la conversión analógico digital.

Tabla 1 Características eléctricas del sistema

\begin{tabular}{llll}
\hline Parámetros & VO & AB & GT \\
\hline Sistema del PPG de referencia $\left(P P G_{D}\right)$ & 9 & $0.5-45$ & 4995 \\
Acelerómetro $(A c c)$ & 3.5 & DC-45 & 1 \\
Sistema del PPG de registro con movimiento $\left(P P G_{R}\right)$ & 9 & $0.5-45$ & 4990 \\
\hline
\end{tabular}

$\mathrm{AB}$ : ancho de banda para la etapa analógica, mostrado en hertz; unidad de medida para frecuencia; GT: factor de ganancia, amplificación total de la señal; adimensional; S: sensibilidad; unidad de medida en milivoltios $(\mathrm{mV})$ sobre g (g constante de gravedad $9.81 \mathrm{mV} / \mathrm{s}^{2}$ ) configurado a $\pm 1.5 \mathrm{~g}$; VO: voltaje de la operación mostrado en voltios; unidad de medida de potencial eléctrico.

\section{Protocolo para registros de señales del pulso fotopletismográfico}

En el modelo de experimentación propuesto se emplearon 2 dedales, uno colocado en el dedo índice de la mano derecha $\left(P P G_{D}\right)$ y el otro en el índice de la mano izquierda $\left(P P G_{R}\right)$. Se obtuvieron registros simultáneos a fin de que cada sujeto sea su propio control y así poder valorar el error con relación a la señal reconstruida por el filtro, una vez procesada fuera de línea. A los participantes del protocolo se les pidió mantener la mano izquierda en estado de reposo durante el primer minuto de adquisición, y posteriormente moverla de manera cuasi-periódica durante los 2 min restantes, mientras que la mano derecha permanecería estática durante los 3 min de adquisición del PPG. Para registrar los movimientos realizados con la mano izquierda, el $\left(P P G_{R}\right)$ tiene incluido el Acc descrito en el apartado anterior. Las señales adquiridas se procesaron posteriormente usando 2 métodos; en el primero de ellos se aplicó directamente la configuración de cancelador de ruido con el algoritmo LMS convencional, y en el segundo se consideró anteponer una etapa de preprocesamiento que realiza las siguientes tareas: calcula y aplica un factor de corrección por regresión lineal, remueve medias y tendencias, y normaliza las señales a la entrada del cancelador de ruido adaptativo.

\section{Descripción del filtro adaptativo como cancelador de ruido}

Los filtros adaptativos tienen la capacidad de ajustar su respuesta impulso, para filtrar las variaciones de interés en la 
señal a procesar, y requieren poco o ningún conocimiento $a$ priori de las características de la señal y del ruido a filtrar. Otra ventaja más de estos filtros es que tienen la capacidad de seguimiento adaptativo de la señal en condiciones no estacionarias, aunque para su funcionamiento precisan de una señal de referencia (en este caso $r(n)$ del Acc) que se correlacione con la señal que debe ser estimada para atenuar el ruido.

Los filtros adaptativos se componen primordialmente de 2 secciones: un filtro digital y un algoritmo de adaptación. Con relación a los filtros digitales existen 2 tipos, los FIR y los IIR. Para este trabajo se optó por un FIR, dado que este tipo de filtros mantiene una respuesta de fase lineal, que es crucial para la aplicación deseada en este trabajo.

En cuanto al algoritmo de adaptación se eligió el algoritmo de LMS, porque la carga computacional demandada es baja, y además es de fácil implementación, ya que no requiere medidas de las funciones de correlación, ni tampoco inversión de la matriz de correlación, como en el caso de los otros algoritmos de adaptación como los de mínimos cuadrados recursivos (recursive least square [RLS]).

Este tipo de filtros pueden tener diferentes configuraciones tales como: predictores lineales, identificadores de sistemas y canceladores de ruido adaptativo ${ }^{18}$. En el procedimiento de filtrado usado en este trabajo se eligió la configuración de cancelador de ruido adaptativo, dado que el ruido que contamina o modifica la morfología de la señal de la fotopletismografía es variante y no permanece en una frecuencia fija, lo que ofrece como resultado, que por momentos se traslape con la señal de interés.

Dos parámetros de suma importancia en este tipo de filtros, que añaden un algoritmo de LMS, son el factor de convergencia y el orden del filtro. El factor de convergencia $\mu$ determina la velocidad de convergencia, el cual es inversamente proporcional al error cuadrático medio mínimo. Debido a la relación inversa entre estos 2 parámetros, existe un compromiso entre ellos, ya que al aumentar uno disminuye el otro, y viceversa. Adicionalmente, $\mu$ está relacionado con el máximo eigenvalor de la matriz de autocorrelación de la señal empleada como referencia, que en este caso es el del $\mathrm{Acc}^{19}$. Para calcular $\mu$, se calcularon los máximos eigenvalores $\lambda_{\max }$ de cada uno de los registros, y posteriormente se promediaron los inversos de estos valores, el valor resultante fue el factor de convergencia empleado que para este caso fue $\mu=0.089$.

Para obtener el orden del filtro se correlacionaron la señal a filtrar y la señal de referencia para cada uno de los registros $^{19}$. La máxima correlación obtenida en promedio fue con un corrimiento de 10 muestras, y bajo este criterio se determinó que el filtro fuera de orden $\mathrm{N}=10$.

En la figura 2A se muestra la configuración del cancelador de ruido implementado para el primer método de procesamiento, donde la señal cruda es procesada haciendo uso del filtro digital tipo FIR de orden $\mathrm{N}=10$, y del algoritmo LMS con un factor de convergencia $\mu$ de 0.089 . En la figura $2 B$ se muestra la configuración para el segundo método propuesto, usando los mismos parámetros de orden y factor de convergencia, pero incluyendo la sección adicional de preprocesamiento para el ajuste de las señales antes de entrar al cancelador de ruido adaptativo. El proceso de filtrado bajo el algoritmo LMS al que es sometida la señal del $P P G_{R}$ es regido primordialmente por las siguientes ecuaciones:

$$
\begin{aligned}
& \ddot{R}(n)=\sum_{m=0}^{M} w_{m}(n) \times r(n-m) \\
& e(n)=\bar{P} P \bar{G}(n)=P P G_{R}(n)-\ddot{R}(n) \\
& w_{m}(n+1)=w_{m}(n)+2 \times \mu \times e(n) \times r(n-m) \\
& 0<\mu<\frac{1}{\lambda_{\max }}
\end{aligned}
$$

donde: $w_{m}$ es un vector de pesos por el que será ponderada la señal de referencia adquirida por el Acc $r(n-m)$; $\bar{P} P \bar{G}(n)$ es el PPG estimado por el procesamiento de filtrado; $\ddot{R}(n)$ es la salida del filtro digital tipo FIR y será el estimado del ruido; $e(n)$ en la configuración de canceladores de ruido corresponde a la señal que estamos aproximando a una señal deseada; $r(n-m)$ es la señal adquirida por el Acc, como señal de referencia para atenuar el ruido; $w_{m}(n+1)$ es la actualización del vector de pesos para cada iteración del filtro; $\mu$ es un parámetro conocido como factor de convergencia; $\lambda_{\max }$ es el máximo eigenvalor de la matriz de autocorrelación de $r(n)$.

\section{Etapa de preprocesamiento para ajuste de las señales del pulso fotopletismográfico y del acelerómetro}

Una vez realizada la captura de los registros de pulso, estos se procesaron fuera de línea con el software Matlab ${ }^{\circledR}$ $\mathrm{R} 2013 \mathrm{~b}^{20}$. El primer proceso al que se someten las señales tanto del $P P G_{D}$ y $P P G_{R}$ es a un ajuste por regresión lineal tomando solo el primer minuto de los registros. Durante este tiempo de registro no existen alteraciones por movimiento, y solo se esperan diferencias menores en amplitud, por lo que se consideró suficiente para este estudio piloto que al ajustar cada pareja de registros se tuviera un coeficiente de determinación $R^{2}>0.8$. Como segundo paso se removió la tendencia lineal que pueden seguir a largo plazo las señales de la fotopletismografía, y que no corresponden al fenómeno del movimiento miocinético. El tercer proceso consistió en eliminar la media de cada una de las señales $\left(P P G_{D}, P P G_{R}\right.$ y $\left.A C c\right)$ para, finalmente, normalizar usando el criterio de máximos de cada una de las señales para evitar las diferencias en amplitud entre los dedales y el Acc al realizar las diferencias de cuadrados en el algoritmo LMS. Todo lo anterior se realizó mediante un script escrito en lenguaje de programación de Matlab ${ }^{\circledR}$.

\section{Valoración estadística del error}

Para valorar el error entre pulsos con ruido y pulsos estimados, mediante los 2 métodos de filtrado, se determinó calcular el error porcentual y la relación señal a ruido. Para calcular el error porcentual $(\% E)$ entre las señales sin movimiento $\left(P P G_{D}\right)$ y las señales estimadas $(\bar{P} P \bar{G})$ se empleó la ecuación 5:

$$
\% E=\left(\frac{P P G-P P G_{D}}{P P G_{D}}\right) \times 100
$$




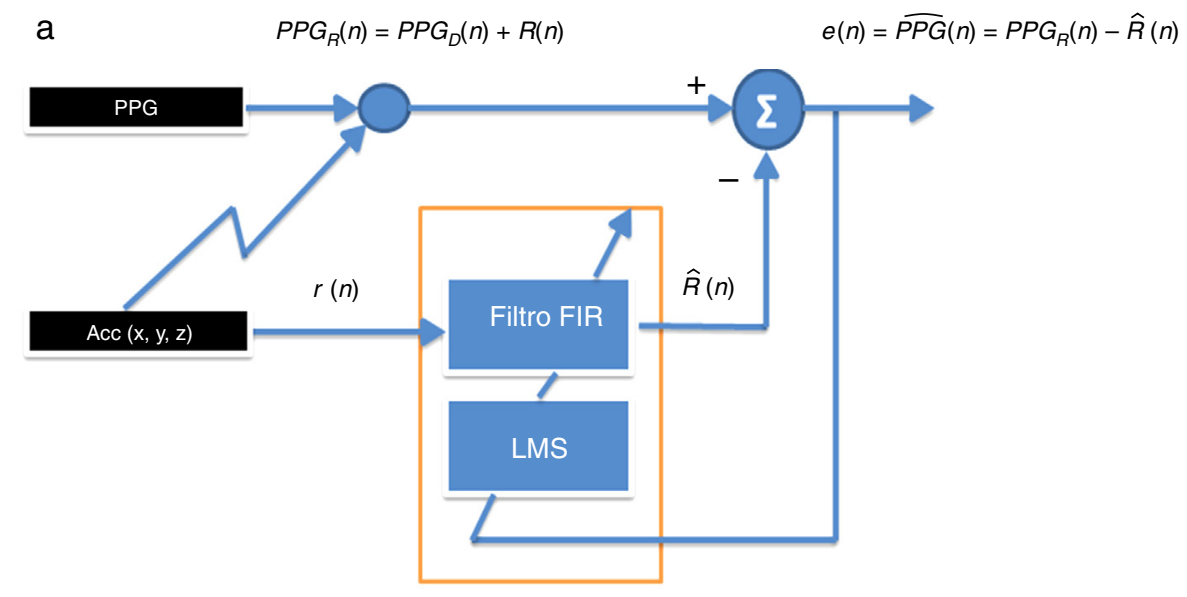

b

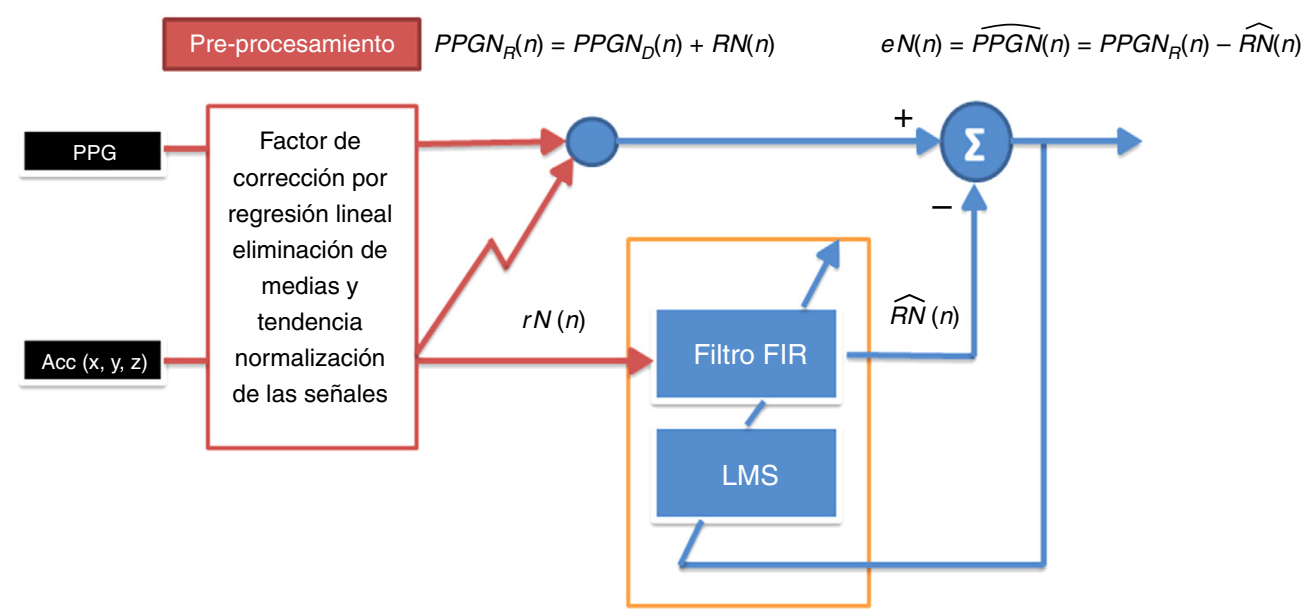

Figura 2 a) Diagrama a bloques, implementado para el primer método, el cual describe la configuración del cancelador de ruido para la atenuación del ruido miocinético en el PPG, donde solo se hace uso del algoritmo LMS. Las siglas empleadas en el diagrama corresponden a las utilizadas en el texto. b) Diagrama a bloques, empleado para el segundo método, el cual describe la composición de la sección adicional de preprocesamiento para el ajuste de las señales que entrarán al cancelador de ruido. En esta figura se adhiere una $\mathrm{N}$ al final de cada sigla indicando que se trata de señales normalizadas.

La distribución de medias de los errores porcentuales se contrastó con una distribución normal bajo la prueba de Kolmogorov-Smirnov, después se compararon las varianzas a fin de verificar si son distintas mediante una prueba de $F$, y para verificar que existen diferencias entre las medias de los errores se realizó una prueba $t$ de Welch. Todas estas pruebas se hicieron con Statistica 8, las que se consideraron significativas bajo un error $\alpha<0.05$.

Se determinó calcular la relación señal a ruido (signalto noise ratio [SNR]) para ambos procedimientos propuestos, con el fin de verificar las semejanzas en potencia entre $P P G_{D}$ y $\widehat{P P G}$. Para el cálculo dedicha relación se ocupó la ecuación 6:

$\operatorname{SNR}(d b)=10 \times \log \left[\frac{P\left(P P G_{D}\right)}{P(\widehat{P P G})}\right]$

donde: $\operatorname{SBR}(d b)$ es la relación señal a ruido dada en decibeles; $P\left(P P G_{D}\right)$ es la potencia de la señal del $P P G_{D}$, sin movimiento; $P\left(\bar{P} P \bar{G}_{D}\right)$ es la potencia de la señal de $\widehat{P P G}$ estimada por el filtro.
En las figura $3 \mathrm{~A}$ y $\mathrm{B}$ se muestra un ejemplo en el cambio entre señales de pulso en el dominio del tiempo, haciéndose evidente las alteraciones del PPG con ruido, y las estimaciones del pulso una vez filtrado bajo ambos métodos propuestos.

\section{Análisis de densidad espectral de potencia}

Otra forma de visualizar las diferencias entre las señales que estamos analizando, además de las que se pueden observar cualitativamente en el dominio del tiempo, es transformándolas al dominio de la frecuencia, donde es posible verificar cuales son los componentes espectrales que deforman la señal de la fotopletismografía, y que no son observados de forma simple en el dominio del tiempo.

En el análisis espectral empleado para comparar las señales se valoró la densidad espectral de potencia (power spectral density [PSD]) utilizando el método de Welch ${ }^{21}$ con $50 \%$ de traslape y una ventana de tipo Hanning de 512 puntos ${ }^{22}$. 


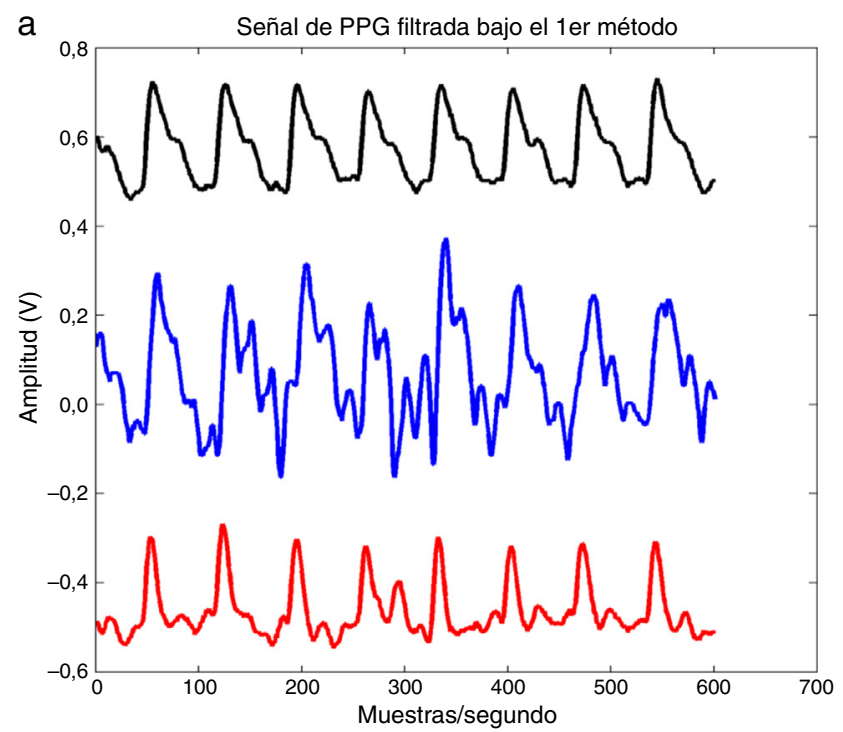

b

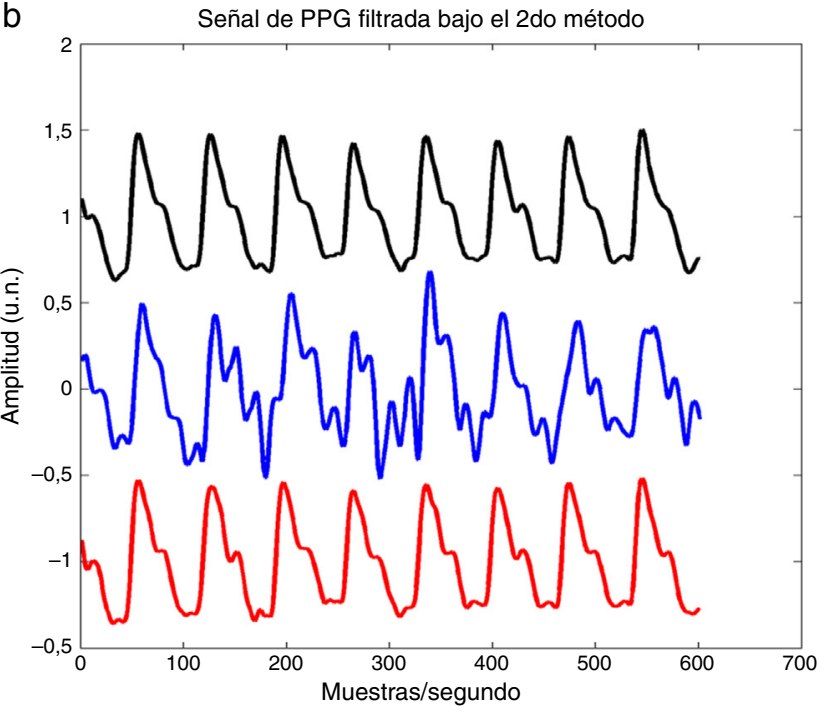

— PPG deseado _ PPG contaminado _ PPG filtrado

Figura 3 a) Inspección visual en los 3 PPG deseado (de referencia) con ruido, y estimado por el filtro bajo el primer método en el dominio del tiempo en uno de los voluntarios estudiados (voluntario 3). Las amplitudes se muestran en unidades normalizadas (u.n.). b) Inspección visual en los 3 PPG deseado (de referencia) con ruido, y estimado por el filtro bajo el segundo método en el dominio del tiempo en uno de los voluntarios estudiados (voluntario 3). Las amplitudes se muestran en unidades normalizadas (u.n.).

\section{Análisis comparativo de identificación de cimas y pies de pulso}

Al aplicar una etapa de preprocesamiento junto con la herramienta de filtrado adaptativo (cancelador de ruido), a las señales del PPG contaminados con artefactos de movimiento, se busca mejorar la calidad de estas señales para aumentar la efectividad de la identificación de cimas y pies de pulso. Para evaluar el porcentaje de identificación correcto se hicieron las detecciones de cimas y pies en $90 \mathrm{~s}$ de registros crudos y filtrados, empleando el criterio de la primera derivada ${ }^{23}$ con las mismas condiciones de umbral de detección para todos los registros.

Para la comparación se tomó en consideración los verdaderos positivos (VP), falsos positivos (FP) y falsos negativos (FN) para obtener valores porcentuales de error, sensibilidad y valor predictivo positivo en la identificación de cimas y pies de pulso, tanto en el registro del PPG de referencia como en el contaminado y en el filtrado.

Para valorar el error, la sensibilidad y el valor predictivo positivo se emplearon las ecuaciones (7), (8) y (9), respectivamente:

$$
\begin{aligned}
& \text { error }=\frac{F P+F N}{V P+F P} \\
& \text { Sens }=\frac{V P}{V P+F N} \\
& V P^{+}=\frac{V P}{V P+F P}
\end{aligned}
$$

donde: error es el porcentaje de error en la identificación de verdaderos positivos; $F N$ es el número de falsos negativos; FP es el número de falsos positivos; Sens es la sensibilidad; $V P$ es el número de verdaderos positivos; $V P^{+}$es el valor predictivo positivo.

\section{Resultados}

Para determinar la pertinencia del uso de la regresión lineal en el preprocesamiento de las señales del PPG, se evaluó el coeficiente de determinación esperando tener $R^{2}>0.8$. Todas las correlaciones realizadas superaron dicho valor. Posteriormente se valoró el grado de atenuación del ruido miocinético bajo 2 métodos de procesamiento de filtrado adaptativo usando un mismo tipo de filtro (FIR) con el mismo orden $(\mathrm{N}=10)$ y factor de convergencia $(\mu=0.089)$, así como con un mismo algoritmo de adaptación (LMS) en condiciones semicontroladas; en el primer método se aplicó directamente el cancelador con el algoritmo LMS, sin procesamientos previos de filtrado o acondicionamiento de la señal, mientras que en el segundo se aplicó una etapa de preprocesamiento previa al cancelador con el algoritmo LMS. Los resultados obtenidos con el primer método mostraron atenuaciones poco apreciables, pues su SNR fue de $15.74 \mathrm{~dB}$, indicando que la señal estimada por el filtro tiene poca potencia con relación a la señal sin movimiento. En cuanto a las diferencias entre la señal $P P G_{D}$ y $\widehat{P P G}$ se obtuvo un error de $22.94 \pm 12.5 \%$ (media \pm desviación estándar). Con el segundo método se obtuvieron atenuaciones de ruido mayores, que permitieron alcanzar una SNR de $0.03 \mathrm{~dB}$, lo que indica que la potencia entre la señal de referencia y la estimada por el filtro son semejantes, permitiendo conseguir un error de $3.72 \pm 2.62 \%$ al evaluar la diferencia entre la señal $P P G_{D}$ y $\widehat{P P G}$.

Al observar errores menores en la segunda metodología, se contrastaron las medias de los errores porcentuales con una distribución normal bajo la prueba de KolmogorovSmirnov, que resultó tener un comportamiento normal. A fin de identificar si las varianzas en los errores porcentuales fueron distintas, y no solo por efecto de aleatoriedad, se procedió a verificarlas mediante una prueba de $F$ que 
Tabla 2 Evaluación del error porcentual, sensibilidad y valor predictivo positivo, en la identificación de cimas del PPG de 10 voluntarios sanos, en registros de $90 \mathrm{~s}$ para cada uno. Resultados obtenidos prefiltrado y posfiltrado

\begin{tabular}{lllllll}
\hline & \% error SR & \% error SF & \% sens SR & \% sens SF & \% VP + SR & \% VP + SF \\
\hline Media & 24.26 & 2.02 & 91.71 & 99.92 & 83.55 & 98.06 \\
DE & 21.11 & 1.88 & 10.77 & 0.25 & 99.66 & 1.95 \\
Min & 0 & 0 & 72.63 & 100 & 61.8 & 93.94 \\
Máx & 59.55 & 6.06 & 100 & 8.21 & 100 & 100 \\
$\Delta$ media & & -22.24 & & & 14.50 \\
\hline
\end{tabular}

\% error SR y \% error SF: error porcentual en la detección de cimas para el PPG ruidoso y filtrado, respectivamente.

\% sens SR y \% sens SF: sensibilidad en la detección de cimas para el PPG ruidoso y filtrado, respectivamente.

$\%$ VP + SR y \% VP + SF: valor predictivo positivo para cimas en el PPG ruidoso y filtrado, respectivamente.

DE: valor porcentual obtenido de la desviación estándar; Máx: valor máximo del conjunto de datos; Media: valor porcentual medio obtenido; Min: valor mínimo del conjunto de datos; $\Delta$ media: diferencia obtenida entre las medias porcentuales del error, la sensibilidad y el valor predictivo positivo, al identificar cimas de pulso antes y después del proceso de filtrado.

resultó significativa, lo que indica que se trata de varianzas distintas, por lo que se procedió arealizar una prueba $t$ de Welch donde se obtuvo una $p=0.008$; este resultado sugiere que las diferencias en los errores porcentuales no corresponden a diferencias por efecto aleatorizado. Comparando los resultados obtenidos bajo ambas metodologías se verificó que la segunda metodología entrega mejoras notables en los porcentajes de error para todos los casos, además que se logró mejorar el tiempo de estimación de mínimo error, que en promedio fue de $25 \mathrm{~s}$, ya que en la primera metodología el tiempo de estimación promedio fue de $32 \mathrm{~s}$. Los resultados de esta segunda metodologías on satisfactorios y útiles para las aplicaciones donde se requiere la recuperación en la morfología de la señal del PPG, por lo que se determinó realizar las comparaciones en las detecciones de cimas y pies de pulso antes y después del proceso de filtrado, solo con el segundo método de filtrado.

Los resultados obtenidos en la identificación de cimas de pulso antes de filtrar los registros tuvieron un error porcentual promedio del 24.26\% (\% Error) calculado con la ecuación 7, y después de filtrar los registros el porcentaje de error disminuyó al 2.02\%; el porcentaje de sensibilidad (\% Sens) aumentó del 91.71 al 99.92\%; y el porcentaje del valor predictivo positivo $\left(\% \mathrm{VP}^{+}\right)$también mejoró incrementando del 83.55 al $\mathbf{9 8 . 0 6 \%}$ de los registros filtrados respecto a los crudos. A partir de estos resultados se obtuvieron diferencias ( $\Delta$ media) entre el error porcentual promedio para el PPG con ruido y filtrados del $-22.24 \%$, entre sensibilidades del 8.21\%, y entre valores del $\mathrm{VP}^{+}$del $14.50 \%$ (tabla 2).

En la detección de los pies de pulso se obtuvo un error porcentual promedio del $48.39 \%$ previo al filtrado, y del $3.77 \%$ posfiltrado. La sensibilidad y el valor predictivo positivo también mejoraron, aumentando del 83.69 al $99.80 \%$ y del 64.75 al $96.61 \%$, respectivamente. Se obtuvieron las siguientes diferencias ( $\Delta$ media) entre el error porcentual promedio para la detección de pies del PPG con ruido y filtrados del $-42.62 \%$, entre sensibilidades del $16.12 \%$, y diferencias del $\mathrm{VP}^{+}$del $31.86 \%$ (tabla 3 ).

La inspección visual de la PSD por el método de Welch, permite observar los cambios que ocurren en el dominio de la frecuencia PSD, mostrando las diferencias entre las densidades espectrales de potencia de la señal contaminada y la filtrada, así como la similitud entre la señal de referencia y la obtenida por el filtro. Como ejemplo, en la figura 4 se
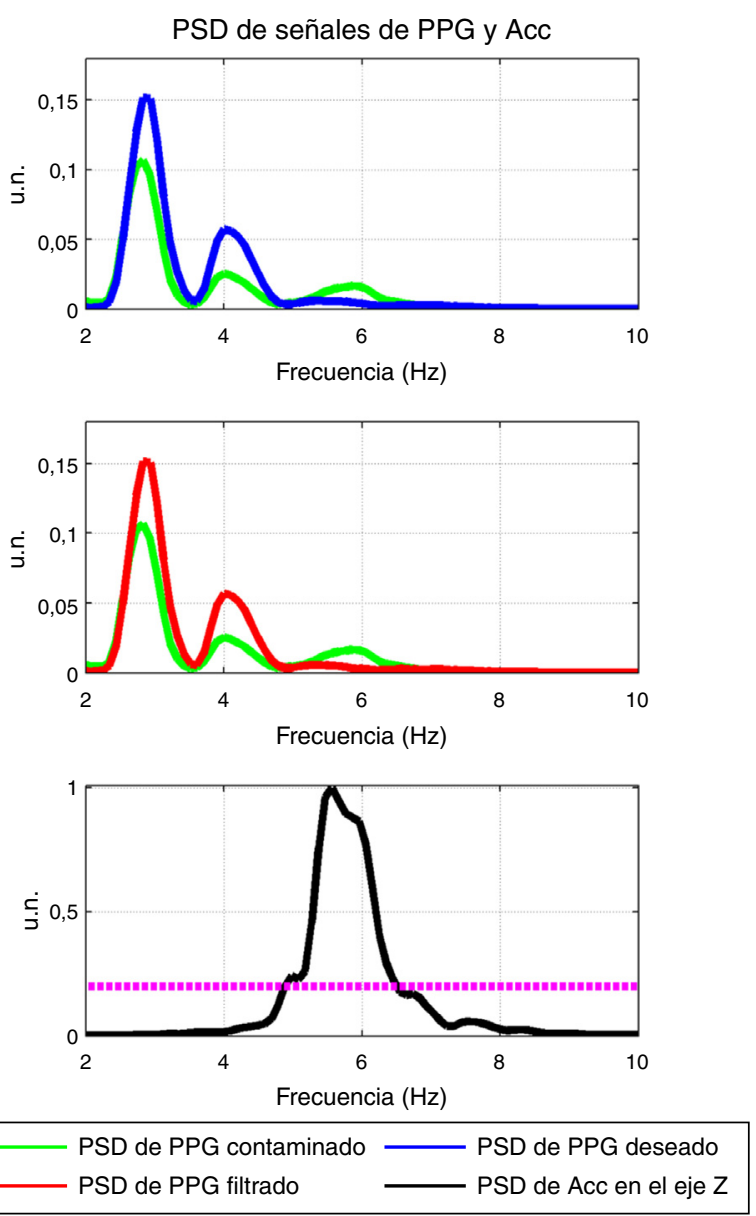

Figura 4 La PSD de la señales del PPG deseado (de referencia), contaminada, filtrada y del Acc en el eje Z, obtenidas por periodograma de Welch con ventana Hanning, con el segundo método de filtrado para el voluntario 3. Las potencias se muestran en unidades normalizadas (u.n.).

ilustra las señales del PPG y del Acc en el PSD. En el panel superior se observan las frecuencias que deforman al PPG y la señal $\widehat{P P G}$, que es la estimación del PPG filtrado, en el panel central se deja ver si se aproximó a la señal de $P P G_{D}$ de 
Tabla 3 Evaluación del error porcentual, sensibilidad y valor predictivo positivo, en la identificación de pies del PPG de 10 voluntarios sanos, en registros de $90 \mathrm{~s}$ para cada uno. Resultados obtenidos prefiltrado y posfiltrado

\begin{tabular}{lllllcc}
\hline & \% error SR & \% error SF & \% sens SR & \% sens SF & VP+SR & VP + SF \\
\hline Media & 48.39 & 3.77 & 83.69 & 99.80 & 64.75 & 96.61 \\
DE & 26.18 & 3.33 & 13.92 & 0.59 & 17.21 & 2.86 \\
Min & 7.81 & 0 & 51.55 & 98.04 & 34.15 & 90.09 \\
Máx & 96.55 & 11.71 & 100 & 100 & 92.19 & 100 \\
$\Delta$ media & & -44.62 & & 16.12 & 31.86 \\
\hline
\end{tabular}

\% error SR y \% error SF: error porcentual en la detección de pies para el PPG ruidoso y filtrado, respectivamente.

$\%$ sens SR y \% sens SF: sensibilidad en la detección de pies para el PPG ruidoso y filtrado, respectivamente.

$\%$ VP + SR y \% VP + SF: valor predictivo positivo para pies para el PPG ruidoso y filtrado, respectivamente.

DE: valor porcentual obtenido de la desviación estándar; Max: valor máximo del conjunto de datos; Media: valor porcentual medio obtenido; Min: valor mínimo del conjunto de datos; $\Delta$ media: diferencia obtenida entre las medias porcentuales del error, la sensibilidad y el valor predictivo positivo, al identificar pies de pulso antes y después del proceso de filtrado.

la mano derecha que fue la empleada como PPG de referencia para la comparación. En el panel inferior de la figura 4 se muestra la PSD del Acc en el eje $Z$, dado que este es el eje que presenta mayor contribución a la deformación del PPG, de acuerdo a los movimientos semicontrolados empleados en el protocolo, con lo que por inspección visual se puede notar en qué banda de frecuencia existe mayor movimiento, y la línea punteada nos indica el umbral que se consideró para evaluar la mayor atenuación del filtro.

Las figura $5 \mathrm{~A}$ y Bmuestran la detección de cimas y pies de pulso en las 3 señales del PPG del voluntario 3.

\section{Discusión}

El filtrado de señales de la fotopletismografía contaminados con artefactos de movimiento, se ha hecho por diversos métodos, las primeras aproximaciones emplearon filtros digitales de coeficientes fijos o también llamados filtros tradicionales, los cuales se configuran como filtros pasa bajos, pasa altos o pasa banda, están limitados al actuar solo sobre un conjunto de frecuencias bien determinadas ${ }^{11}$. Otra forma en que han filtrado estas señales es mediante filtros de promedio móvil; sin embargo, este procedimiento funciona bien solo para una gama limitada de artefactos, presentando efectos no deseados debido a la segmentación que se realiza bajo esta técnica ${ }^{24}$. En la práctica, el ruido debido al movimiento miocinético se puede reducir con éxito, con el uso de filtros adaptativos, manteniendo a la vista la simplicidad en la implementación, además de las ventajas como son un menor tiempo de respuesta y la capacidad de seguimiento de las señales implicadas para continuar la transformación bajo condiciones de movimiento ${ }^{11}$.

Los resultados obtenidos en el desarrollo de este trabajo muestran que es posible aumentar la efectividad de la detección de cimas y pies en señales de PPG deformados por ruido miocinético, al emplear técnicas de filtrado adaptativo que permiten remover los efectos de los artefactos de movimiento, y reconstruir señales del PPG altamente semejantes a una señal que no presente deformaciones. Los resultados obtenidos mostraron incrementos de detección de un $22.24 \%$ para cimas y de un $42.62 \%$ para pies, lo que indica que la mayor contribución se atribuye en la mejora de la identificación de pies de pulso.
Tener una mayor efectividad en la detección de cimas y pies de pulso tiene como implicación una mayor confianza en la valoración médica, pues la identificación de puntos notables del pulso es útil para el cálculo de la frecuencia cardiaca, o en periodos más extendidos, estudios como la variabilidad de la frecuencia cardiaca ${ }^{25}$, también es útil para la correcta medición de la saturación de oxígeno por el método de oximetría 26,27 . En cuanto a la correcta recuperación en la morfología de la señal del PPG, esta podría ayudar a determinar si existen o no problemas de rigidez arterial o fuertes cambios de volumen sanguíneo como la vasoconstricción o vasodilatación ${ }^{16}$.

Obtener un bajo porcentaje de error entre las señales de referencia y recuperada, junto con una alta efectividad de detección de puntos notables haciendo uso de algoritmos de baja complejidad computacional, permiten tener la posibilidad de implementar esta metodología en dispositivos embebidos que podrían ser comunicados con dispositivos móviles.

El diseño del sistema presentado en este trabajo tiene limitaciones cuando las condiciones de ruido sobrepasan los cambios de aceleración del Acc, pues fue diseñado para aceleraciones con rango de $\pm 1.5 \mathrm{~g}$ siendo $g=9.81 \mathrm{~m} / \mathrm{s}^{2}$. Cuando la aceleración es mayor no es posible registrarla correctamente $y$, en consecuencia, el sistema no actúa como se espera.

Como trabajo futuro, el sistema podría aumentar sus capacidades en hardware al ser reajustado para detectar mayores cambios de aceleración, lo que le permitiría registrar artefactos de movimiento debidos a incrementos de fuerza. Se propone también que el procesamiento sea en línea, usando dispositivos embebidos de bajas prestaciones, pues el desarrollo de este sistema con algoritmos de baja complejidad computacional lo hacen óptimo para este tipo de implementaciones.

El sistema actual podría usarse en pacientes con daños motores o neurodegenerativos como el mal de Parkinson, aunque su utilización puede ser extendida a más aplicaciones, haciendo las modificaciones de hardware correspondientes, podría tener posibles aplicaciones en el área médico-deportiva usándolo en atletas en entrenamiento a fin de evaluar distintas variables fisiológicas que permitan hacer una valoración médica del deportista usando esta técnica. 


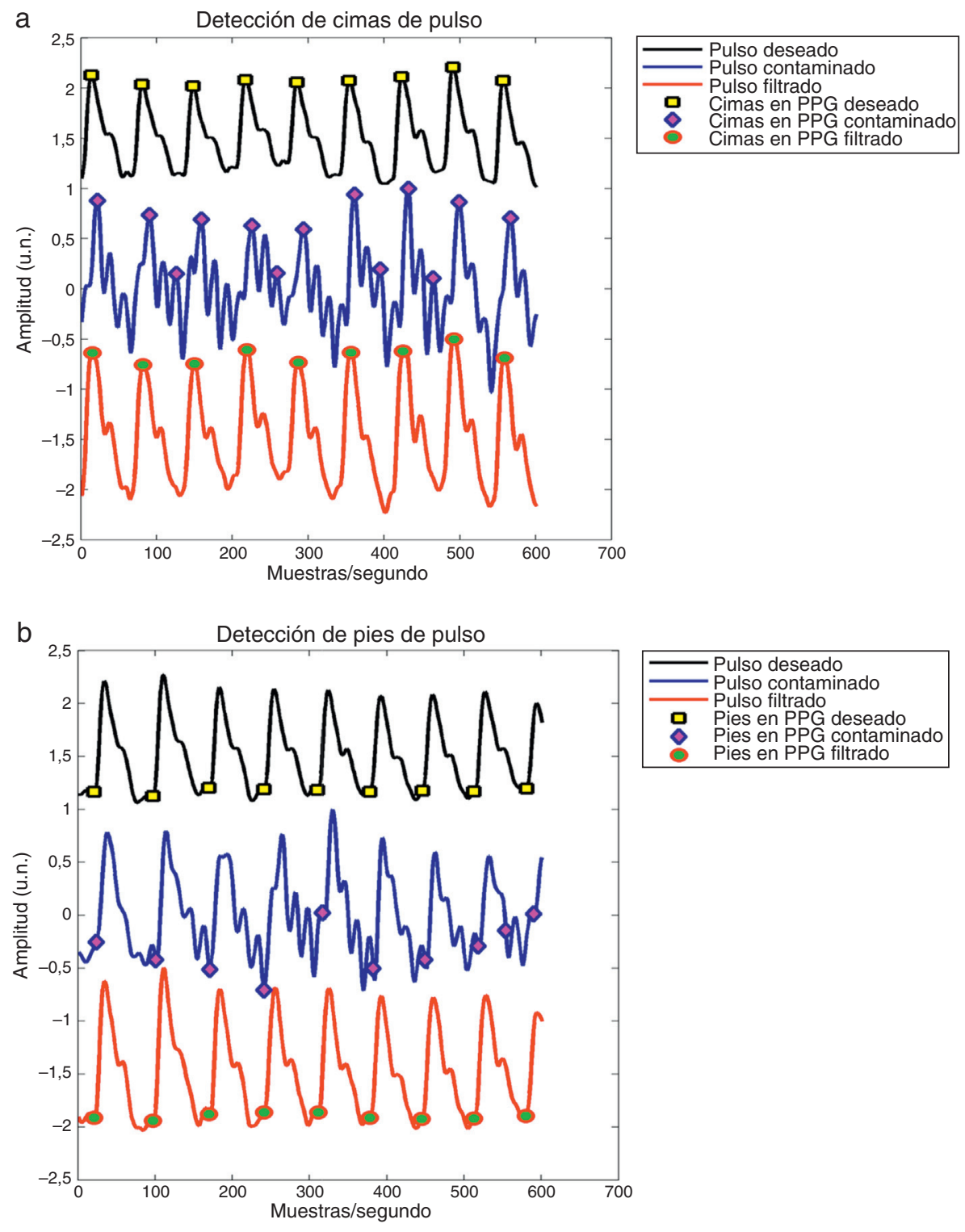

Figura 5 a) Ejemplo de identificación de cimas en el PPG de referencia, contaminadas y filtradas. En esta figura se muestra la aparición de falsos positivos para las cimas del pulso contaminado. Las amplitudes de los registros se muestran en unidades normalizadas (u.n.). b) Ejemplo de identificación de pies en el PPG de referencia, contaminadas y filtradas. En esta figura se muestra la aparición de falsos positivos para los pies del pulso contaminado. Las amplitudes de los registros se muestran en unidades normalizadas (u.n.).

Por otro lado, también existe la posibilidad de desarrollar sistemas de la fotopletismografía inalámbricos que se comuniquen con el ordenador, donde se podrían implementar algoritmos de mayor complejidad computacional, a fin de lograr mejores resultados en cuanto a similitud de las señales, junto con un menor tiempo de recuperación de la señal, pues el algoritmo empleado es lento en su velocidad de convergencia comparado con otros como el filtro de Kalman ${ }^{28}$.

\section{Conclusiones}

Por medio del análisis estadístico y del análisis de la densidad espectral sobre las señales del PPG, se muestra que es posible mejorar la calidad de las señales del PPG sometidas a ruido miocinético, toda vez que se incorpore un elemento de detección de movimiento Acc en el dedal de la fotopletismografía, para así poder aplicar técnicas de filtrado adaptativo (cancelador de ruido).

El método de filtrado adaptativo con la etapa de preprocesamiento demostró ofrecer mejores resultados, manteniendo una baja carga computacional con el algoritmo LMS.

Recuperar las señales del PPG con un bajo porcentaje de error, permitió mejorar la detección de cimas y pies de pulso, siendo estos últimos donde se obtuvo una mejora mayor en su detección. Todo esto tiene como implicación gozar de una mayor confianza en la valoración médica por medio de la técnica de la fotopletismografía. 


\section{Responsabilidades éticas}

Protección de personas y animales. Los autores declaran que para esta investigación no se han realizado experimentos en seres humanos ni en animales.

Confidencialidad de los datos. Los autores declaran que han seguido los protocolos de su centro de trabajo sobre la publicación de datos de pacientes.

Derecho a la privacidad y consentimiento informado. Los autores declaran que en este artículo no aparecen datos de pacientes.

\section{Financiación}

No se recibió patrocinio de ningún tipo para llevar a cabo este artículo.

\section{Conflicto de intereses}

Los autores declaran no tener ningún conflicto de intereses.

\section{Bibliografía}

1. Frenk J. Globalization, health, and the role of telemedicine. Telemed J E Health. 2005;11:291-5.

2. Dabaghi-Richerand A, Chávarri A, Torres-Gómez A. Telemedicina en México. An Med Asoc Med Hosp ABC. 2012;57:353-7.

3. Becerra-Luna B, Dávila-García R, Salgado-Rodríguez P, et al. Monitor de señales de electrocardiografía y frecuencia cardiaca mediante un teléfono móvil con el protocolo de comunicación Bluetooth. Arch Cardiol Mex. 2012;82:197-203.

4. Allen J. Photoplethysmography and its application in clinical physiological measurement. Physiol Meas. 2007;28:R1-39.

5. Elgendi M, Norton I, Brearley M, et al. Systolic peak detection in acceleration photoplethysmograms measured from emergency responders in tropical conditions. PLoS One. 2013;8:e76585.

6. Zhang Z, Pi Z, Liu B. TROIKA: a general framework for heart rate monitoring using wrist-type photoplethysmographic signals during intensive physical exercise. IEEE Trans Biomed Eng. 2015;62:522-31.

7. Allen J, Murray A. Age-related changes in the characteristics of the photoplethysmographic pulse shape at various body sites. Physiol Meas. 2003;24:297-307.

8. Sommermeyer D, Zou D, Ficker JH, et al. Detection of cardiovascular risk from photoplethysmography signal using a matching pursuit algorithm. Med Biol Eng Comput. 2015:1-11.

9. Thakor NV. Applications of adaptive filtering to ECG analysis: Noise cancellation and arrhythmia detection. IEEE Trans Biomed Eng. 1991;38:785-94.

10. Becerra-Luna B, Sánchez JC, Martińez-Memije R, et al. Photoplethysmographic fingertip with miokinetic noise attenuation using an accelerometer and adaptive filtering techniques. Health Care Exchanges (PAHCE). 2013:1-5.
11. Ram MR, Madhav KV, Krishna EH, et al. A novel approach for motion artifact reduction in PPG signals based on AS-LMS adaptive filter. IEEE Trans Instrum Meas. 2011;61:1445-57.

12. Solosenko A, Petrenas A, Marozas V. Photoplethysmographybased method for automatic detection of premature ventricular contractions. IEEE Trans Biomed Circuits Syst. 2015;9: 662-9.

13. Cassani R, Mejía P, Tavares JA, et al. Adaptive filtering for respiration influence reduction on heart rate variability. 8th International Conference Electrical Engineering Computing Science and Automatic Control (CCE). 2011. p. 1-5.

14. Widrow B, Glover J Jr, McCool J, et al. Adaptive noise cancelling: Principles and applications. IEEE Proceedings. 1975;63:1692-716.

15. Haykin S, Widrow B. Least mean square adaptive filters. New Jersey: John Wiley and Sons Inc; 2003.

16. Sánchez-Torres G, Infante-Vázquez O, Sánchez-Miranda G, et al. Mechanical factors of cardiovascular risk in systemic arterial hypertension. A new sign of arterial rigidity. Arch Cardiol Mex. 2003;73:261-70.

17. Introducing TracerDAQ, Help Revision 18, Measurement Computing. [consultado Jul 2015] Disponible en: http://www.mccdaq.com/pdfs/manuals/TracerDAQHelp.zip

18. Avalos JG, Sánchez JC, Velázquez J. Applications of adaptive filtering. En: García L, editor. Adaptive Filtering Applications, 1. InTech; 2011. p. 3-20.

19. Akay M. Adaptive noise canceling. En: Akay M, editor. Biomedical signal processing Orlando. Florida: Academic Press; 1994. p. 139-45.

20. MathWorks, Inc QuickStart Matlab Primer R2015b. [consultado Mar 2015] Disponible en: https://www.mathworks.com/help/ pdf_doc/matlab/getstart.pdf

21. Welch PD. The use of fast Fourier transform for the estimation of power spectra: A method base on time averaging over short, modified periodograms. IEEE Trans Audio and Electroaccoust. 1967; 15:70-3.

22. Gautam JK, Kumar A, Saxena R. On the modified BartlettHanning window (family). IEEE Trans Signal Processing. 1996;44:2098-102.

23. Li BN, Dong MC, Vai MI. On an automatic delineator for arterial blood pressure waveforms. Biomed Signal Process Control. 2009;5:76-81.

24. Lee HW, Lee JW, Jung WG, et al. The periodic moving average filter for removing motion artifacts from PPG signals. Int J Control Autom. 2007;5:701-6.

25. Yang GL, Taylor JA, Stein JF. A comparison of photoplethysmography and ECG recording to analyse heart rate variability in healthy subjects. J Med Eng Technol. 2009;33: 634-41.

26. Cahan C, Decker MJ, Hoekje PL, et al. Agreement between noninvasive oximetric values for oxygen saturation. Chest. 1990;97:814-9.

27. Chan ED, Chan MM, Chan MM. Pulse oximetry: Understanding its basic principles facilitates appreciation of its limitations. Respir Med. 2013;107:789-99.

28. Vullings R, Vries B, Bergmans JWM. An adaptive Kalman filter for ECG signal enhancement. IEEE Trans Biomed Eng. 2011;58:1094-103. 